\section{In-vitro-Testsystem für spezifisches IgE}

a aboruntersuchungen sind ein unverzichtbarer Bestandteil der allergologischen Diagnostik. Im Gegensatz zu den klinischen Testverfahren haben sie den Vorteil einer genaueren Kontrolle der Sensitivität und Spezifität, belasten den Patienten weniger und sind auch bei hochgradiger Sensibilisierung ohne Gefahr für den Patienten durchführbar. Zwar analysieren In-vitro-Testungen, z.B. gegen spezifisches IgE, nur einen Teilabschnitt der im Patienten ablaufenden allergischen Reaktionskaskade und erlauben keine direkte Aussage über die Aktualität der klinischen Symptomatik - doch geben sie einen sicheren Hinweis zum Sensibilisierungsmuster des Patienten. In Hinblick auf den Nachweis einer allergischen Sensibilisierung eignen sich In-vitro-Testungen angesichts der hohen Qualität auch für Screening- und Reihenuntersuchungen.

Ein neues qualitatives In-vitro-System zum Nachweis spezifischer IgE-Antikörper in Humanseren gegenüber Aeroallergenen bietet die Basisversion des AllergyScreen, das über die Firma HAL in Deutschland vertrieben wird. Es arbeitet ähnlich der bekannten Technologie des Dot-Blottings. Getestet werden können mit diesem System Sensibilisierungen gegen die Hausstaubmilben Dermatophagoides pteronyssinus und farinae, gegen Baumpollen wie z.B. Birke, gegen Gräser- und Beifußpollen, Katzen-, Pferde- und Hundeepithelien sowie Alternariasporen. Prinzipiell ist das System aber auch für andere Allergenkombinationen geeignet. Für die Analyse sind $400 \mu \mathrm{l}$ Serum notwendig, die Auswertung erfolgt optisch-visuell ohne technisches Zubehör anhand von vier Farbklassen. Der technische und personelle Aufwand ist gering: Für zehn Membranstreifen werden 5 Minuten Arbeitszeit veranschlagt, wobei die Testdurchführung sehr einfach ist. Die Inkubationszeiten sind mit insgesamt 130 Minuten (bei Verwendung des optionalen Schüttkers) kurz, die Kosten niedrig. Das CE-zertifizierte Medizinprodukt ist in Kitgrößen für 10 Bestimmungen erhältlich.

In einer Untersuchung von 110 Seren von Patienten mit einer allergischen Atemwegserkrankung kamen die Ergebnisse, die mit den Basisallergenen des AllergyScreen erhalten wurden, sehr nahe an die Sensitivität und Spezifität des CAP-Systems heran. Lipämische und hämolytische Seren haben keinen Einfluss auf die Testergebnisse.

Nach Informationen der Mediwiss Analytic, 2000.

\title{
Mehr Compliance durch Pulverinhalator
}

nhalative Kortikoide werden auch in absehbarer Zeit unverzichtbarer Bestandteil der Therapie des leichten bis schweren Asthma bronchiale bleiben. Als antientzündliche Basistherapie sind sie in den Therapieempfehlungen der deutschen Atemwegsliga verankert. Als Applikationsform am weitesten verbreitet sind derzeit noch die Dosieraerosole, wie Prof. Dr. Gert Kunkel (Berlin) auf einem Satelliten-Symposium mitteilte. Allerdings weisen diese Geräte gewisse Nachteile auf. So ist z.B. die Koordination zwischen dem Auslösen des Aerosols und dem gleichzeitigen Einatmen schwierig, was dazu führt, dass 30 bis $40 \%$ der Patienten ohne ausreichende Schulung nicht zurecht kommen.

Nicht zuletzt das Verbot von FCKW als Treibmittel hat die Entwicklung von Pulverinhalatoren vorangetrieben. „Bei Neuentwicklungen steht die Compliance als Forderung ganz oben an“, erklärte Kunkel. Hierfür müssen eine Reihe von Anforderungen erfüllt werden: einfache und sichere Handhabung, Inhalationskontrolle zum Beispiel durch ein Zählwerk, guter Ge- schmack. Für Patienten mit schwerem Asthma, aber auch für Kinder, ist besonders wichtig, dass die Deposition in der Lunge bereits bei einem möglichst niedrigen inspiratorischen Fluss erfolgt. Außerdem sollte es auch möglich sein, ein und dasselbe Gerät für verschiedene Asthma-Wirkstoffe einzusetzen.

Der Novolizer ${ }^{\circledR}$ erfülle im Prinzip die genannten Kriterien, bemerkte Kunkel. Er ist relativ umweltfreundlich: Nur die Nachfüllpatrone, mit der das System ausgestattet ist, landet nach Gebrauch im Mülleimer. Über ein Zählwerk kann der Patient erkennen, wie viele von den 200 Einzeldosen sich noch im Gerät befinden. Drei Merkmale bestätigen ihm, dass er erfolgreich inhaliert hat: Farbwechsel im Kontrollfenster, Klickgeräusch und süßer Geschmack des Trägerstoffs Laktose.

In zwei Studien wurden Wirksamkeit, Handhabung und Sicherheit des Novolizers ${ }^{\circledR}$ mit Dosieraerosolen, einmal mit Salbutamol und einmal mit Budesonid, verglichen. In der ersten Studie mit Salbutamol über vier Wochen verbesserte sich das $\mathrm{FEV}_{1}$ in beiden Patientengrup- pen im Mittel um etwa 20\%. In einem Fragebogen äußerten $78 \%$ der AsthmaPatienten, die vorher ein Dosieraerosol verwendet hatten, dass sie in Zukunft den neuen Pulverinhalator verwenden würden. Von den Patienten, die bereits vorher einen Pulverinhalator gebrauchten, präferierten $87 \%$ das neu entwickelte Gerät.

In einer weiteren Untersuchung wurde Novopulmon ${ }^{\circledR} 200$, also der Novolizer $^{\circledR}$ mit Budesonid, einem Referenz-Gerät bei über 300 Patienten mit mildem bis mäßig starkem Asthma bronchiale gegenübergestellt. In einer Dosierung von zweimal täglich $200 \mu \mathrm{g}$ über 12 Wochen ergab sich kein signifikanter Unterschied im Verlauf von $\mathrm{FEV}_{1}$. Auch die Gesamtbeurteilung fiel für beide Systeme gleich aus, berichtete Kunkel. Viele Patienten allerdings würden den Pulverinhalator in der Zukunft bevorzugen, vor allem da er sich einfach und diskret bedienen lässt. $\quad m b$

Satelliten-Symposium „Gute Patientencompliance gleich hoher Therapieerfolg Stimmt die Gleichung?" anlässlich des 42. Kongresses der Deutschen Gesellschaft für Pneumologie in Jena, 2001, Veranstalter: Asta Medica. 